\title{
Mechanism of human somatic reprogramming to iPS cell
}

\author{
Rika Teshigawara, Junkwon Cho, Masahiro Kameda and Takashi Tada
}

Somatic reprogramming to induced pluripotent stem cells (iPSC) was realized in the year 2006 in mice, and in 2007 in humans, by transiently forced expression of a combination of exogenous transcription factors. Human and mouse iPSCs are distinctly reprogrammed into a 'primed' and a 'naïve' state, respectively. In the last decade, puzzle pieces of somatic reprogramming have been collected with difficulty. Collectively, dissecting reprogramming events and identification of the hallmark of sequentially activated/silenced genes have revealed mouse somatic reprogramming in fragments, but there is a long way to go toward understanding the molecular mechanisms of human somatic reprogramming, even with developing technologies. Recently, an established human intermediately reprogrammed stem cell (iRSC) line, which has paused reprogramming at the endogenous OCT4-negative/exogenous transgene-positive pre-MET (mesenchymal-toepithelial-transition) stage can resume reprogramming into endogenous OCT4-positive iPSCs only by change of culture conditions. Genome-editing-mediated visualization of endogenous OCT4 activity with GFP in living iRSCs demonstrates the timing of OCT4 activation and entry to MET in the reprogramming toward iPSCs. Applications of genome-editing technology to pluripotent stem cells will reshape our approaches for exploring molecular mechanisms.

Laboratory Investigation (2017) 97, 1152-1157; doi:10.1038/labinvest.2017.56; published online 22 May 2017

\section{REPROGRAMMING OF SOMATIC CELLS}

Reprogramming somatic cells into induced pluripotent stem cells (iPSCs), which possess unique properties of self-renewal and differentiation into multiple cell lineages, is achieved by transduction using a defined set of transcription factors: Oct4 (Pou5f1), Sox2, Klf4, and c-Myc (OSKM) in mice, ${ }^{1,2}$ and humans. ${ }^{3,4}$ The success of iPSC generation opens a way to produce patient-specific pluripotent stem cells with less ethical issues than embryonic stem cells (ESCs) generated from fertilized pre-implantation embryos. Personalized iPSCs are expected to contribute to the exploration of cure and cause of diseases, drug screening, and tailor-made regenerative medicine. iPSC generation methodology has improved with different delivery systems, including non-integrating vectors, deletion after integration, DNA-free transduction, and chemical induction. ${ }^{5-11}$ Furthermore, novel approaches for iPSC production have been developed, including combinations of alternative transcription factors. ${ }^{12}$ In addition to those, reprogramming-susceptible cell types have been identified. ${ }^{13-15}$ Even with studious effort for methodological and technical improvements, the efficiency of reprogramming remains $\sim 0.1 \%$ in humans, and $\sim 1.0 \%$ in mice. ${ }^{16}$ Mechanisms of somatic reprogramming have important implications for iPSC applications. Furthermore, iPSCs could be of great use in exploring molecular mechanisms of many diseases and embryonic development as models. However, the low efficiency and stochastic nature of reprogramming hinders the understanding of reprogramming mechanisms.

\section{MECHANISMS OF REPROGRAMMING IN MICE}

In mice, the specific order of reprogramming events has been determined as (i) activation of alkaline phosphatase, (ii) silencing of somatic-specific expression, (iii) expression of SSEA1, and (iv) progressive silencing of exogenous genes with concomitant upregulation of endogenous Oct4 and $\operatorname{Nanog}^{17-19}$ (Figure 1a). Nanog is a key player of the stem cell regulatory network critical for acquiring a pluripotent state. ${ }^{20}$ EpCAM, c-Kit, and PECAM1 were identified as other surface markers of early, intermediate, and late genes of SSEA1-positive cells, respectively. ${ }^{21,22}$ Information on roadblock genes during reprogramming and stage-specific markers for enrichment of intermediately reprogrammed cells prone to forming iPSCs is being accumulated using several advanced technologies.

In addition to the marker genes, pluripotency-associated mmu (Mus Musculus) -microRNAs (miRNAs) are sequentially expressed, and implicated with induction, maturation, and stabilization of unique characteristics of PSCs $^{23-25}$ (Figure 1a). In chromatin reprogramming, pioneer 


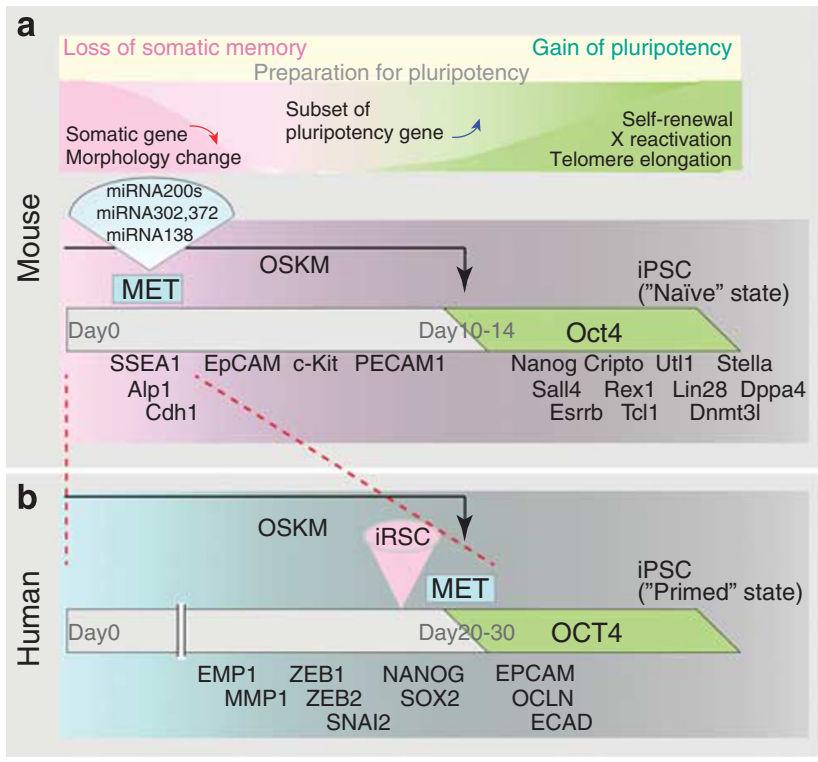

Figure 1 Sequential events, changes of gene and microRNA expression in reprogramming mouse (a) and human (b) somatic cells to induced pluripotent cells (iPSCs). Mesenchymal-to-epithelial transition (MET) occurs at differential timing in the road of reprogramming. Duration of expression of exogenous reprogramming factors, Oct4, Sox2, Klf4, and c-Myc (OSKM) is indicated by black arrow. Expression of endogenous Oct4/OCT4 is indicated by the green rectangle. Derivation of timing of intermediately reprogrammed stem cells (iRSCs) in the reprogramming process is shown by the pink triangle in humans (b).

transcription factors bind directly to condensed chromatin and elicit a series of chromatin remodeling events, including histone modification of $\mathrm{H} 3$ lysine 4 and 27 (ref. 26) that lead to opening of chromatin during cell division, which creates opened chromatin situation that other transcription factors are readily accessible. ${ }^{27}$ In cell cycle reprogramming, rapid proliferation with a characteristic cell cycle structure of short G1- and G2-gap phases is signature to iPSCs accompanying self-renewal and pluripotency. ${ }^{28}$

MET (mesenchymal-to-epithelial transition), which is the hallmark critical event toward the derivation of iPSCs from mouse embryonic fibroblasts (MEFs), occurs at an early stage of reprogramming. ${ }^{23,29,30}$ MET is characterized by upregulation of epithelial genes, E-Cadherin, Cdh1, Epcam, and epithelialassociated mmu-miRNA-200 family, and down-regulation of mesenchymal genes, Snail1/2, Zeb1/2, and N-Cadherin. ${ }^{31-33}$ Exogenous Oct 4 and Sox 2 bind to the promoter regions of mmu-miRNA-141/200c and the mmu-miRNA-200a/b/429 cluster, respectively, and induce transcription activation of the mmu-miRNA-200 family (miRNA-200s). ${ }^{29}$

MET is driven by a strong bone morphogenetic protein (BMP) response through induction of mmu-miRNA-200s and 205 according to the BMP-miRNA-MET pathway. ${ }^{23}$ Repression of mmu-miRNA-200s with specific inhibitors results in repression of MET and iPSC generation. Furthermore, the effects of mmu-miRNA-200s and 205 were blocked by Zeb2 overexpression. Collectively, the mmu-miRNA-200/ Zeb2 pathway critically functions in promoting MET at the early stage of somatic reprogramming. ${ }^{29,34}$ Moreover, MET is controlled under the orchestrated regulation of epigenetic modification modulated, in part, by $\mathrm{H} 3 \mathrm{~K} 36$ demethylases Jhdm1a/1b ${ }^{35}$ and H3K79 methylase Dot1L. ${ }^{36}$ Consequently, MET accompanied by changes in morphology from the somatic to pluripotent type cell induces expression pattern changes in several thousands of genes. ${ }^{23,24}$

\section{MECHANISMS OF REPROGRAMMING IN HUMANS}

Human iPSC colonies exhibit characteristic flat-shaped morphology, which is clearly distinct from mouse iPSC colonies that exhibit bowl-shaped morphology. Mouse iPSCs are reprogrammed into a 'naïve' state similar to the state of mouse embryonic stem cells (ESCs), whereas human iPSCs are in a 'primed' state similar to the state of human ESCs, and mouse Epistem cells (EpiSCs) ${ }^{37,38}$ (Figure 1b). Mouse ESCs, but not mouse EpiSCs, are germline-competent in blastocystinjection-mediated chimeras. Notably, 'primed'-state human and 'naïve'-state mouse iPSCs make differential responses in mouse ground state culture conditions with N2B27+2i+LIF medium. ${ }^{39,40}$ Distinct pluripotent states between human and mouse iPSCs are also demonstrated by X-chromosome reactivation of female somatic cell reprogramming. ${ }^{41}$ Collectively, the final cell fate by somatic reprogramming through forced expression of the same exogenous OSKM transcription factors is distinctive between humans and mice (Figure 1a and $b$ ). Thus, it is predicted that parts of the reprogramming process are shared with humans and mice, whereas others are unique to humans or mice.

Indeed, partially diverged interactions of pluripotencyassociated miRNAs and the target mRNAs between humans and mice have been summarized. ${ }^{25}$ This is consistent with the divergence of sequential reprogramming events between humans and mice. In mice, MET occurs early in reprogramming of MEFs to iPSCs, which precedes the activation of endogenous Oct4 $;^{21,23}$ however, in humans, MET occurs at a later stage of reprogramming with the same timing of endogenous OCT4 activation (Figures 1a and b). It is likely that MET is a checkpoint for entry into a 'primed' state of pluripotency, whereas activation of an OCT4/Oct4 is a key step for commitment to further cellular reprogramming through composing OCT4/Oct4-induced pluripotency molecular network. In this context, human iPSCs acquired OCT4induced pluripotency under a 'primed' state prior to conversion to a 'naïve' state. This implies that, in mice, a 'primed 'state is generated at a much earlier stage, with additional steps required prior to Oct4-induced pluripotency. MET is an event separable from activation of endogenous OCT4/Oct4, as shown by differential timing of entry to MET between human and mouse reprogramming.

The generation of human 'naïve' iPSCs, which demonstrated molecular characteristics and functional properties similar to mouse ESCs/iPSCs, was reported with the 
chemically defined culture conditions, NHSM (naïve human stem cell medium). ${ }^{42}$ Conversion from a 'primed' state to a 'naïve' state is facilitated by forced expression of exogenous Klf4 under ground state culture conditions in mice. ${ }^{43}$ Furthermore, it was revealed that the pluripotencyassociated hsa (Homo sapiens) -miRNA-290/302 family of microRNAs regulates the transition of ESCs from a 'naïve' to 'primed' state of pluripotency. ${ }^{44}$ However, in humans, mechanisms involved in 'primed-to-naïve' conversion are largely unknown.

In human ESCs, OCT4, SOX2, and NANOG, which play essential roles in somatic reprogramming to iPSCs, co-occupy a substantial portion of more than 300 target genes with collaboration to form regulatory circuitry consisting of autoregulatory and feedforward loops, ${ }^{45}$ suggesting that appropriate transcription of OCT4, SOX2, and NANOG is required to stabilize a pluripotency molecular network for facilitating the maturation of the somatic reprogramming process toward iPSC generation. Prior to stabilization of the pluripotency network through MET and endogenous OCT4 activation in human reprogrammed cells, a 'primed' pluripotency competent state may be induced by forced expression of $c-M y c$ and Klf4 as demonstrated by mouse reprogramming cells. ${ }^{30}$ Recently, several 'naïve'-specific, but not 'primed'-specific, cell surface marker proteins were demonstrated by comprehensive profiling of cell surface proteins by flow cytometry in 'naïve' and 'primed' human pluripotent stem cells (PSCs). ${ }^{46}$ It is unclear whether stabilization of human iPSCs under a 'primed' state but not a 'naïve' state resulted from passing through a transient 'naïve' state during reprogramming. Newly identified 'naïve'-specific marker proteins could facilitate to define the human pre-iPSC and iPSC state in the progress of somatic reprogramming.

\section{EXPLORING REPROGRAMMING MECHANISMS WITH AN INTERMEDIATELY REPROGRAMMED STEM CELL LINE}

In mice, intermediately reprogrammed cells characterized by silencing somatic genes, activated SSEA1, and the potential of conversion to iPSCs were predicted as a transient cell population, whereas partially reprogrammed cells

a
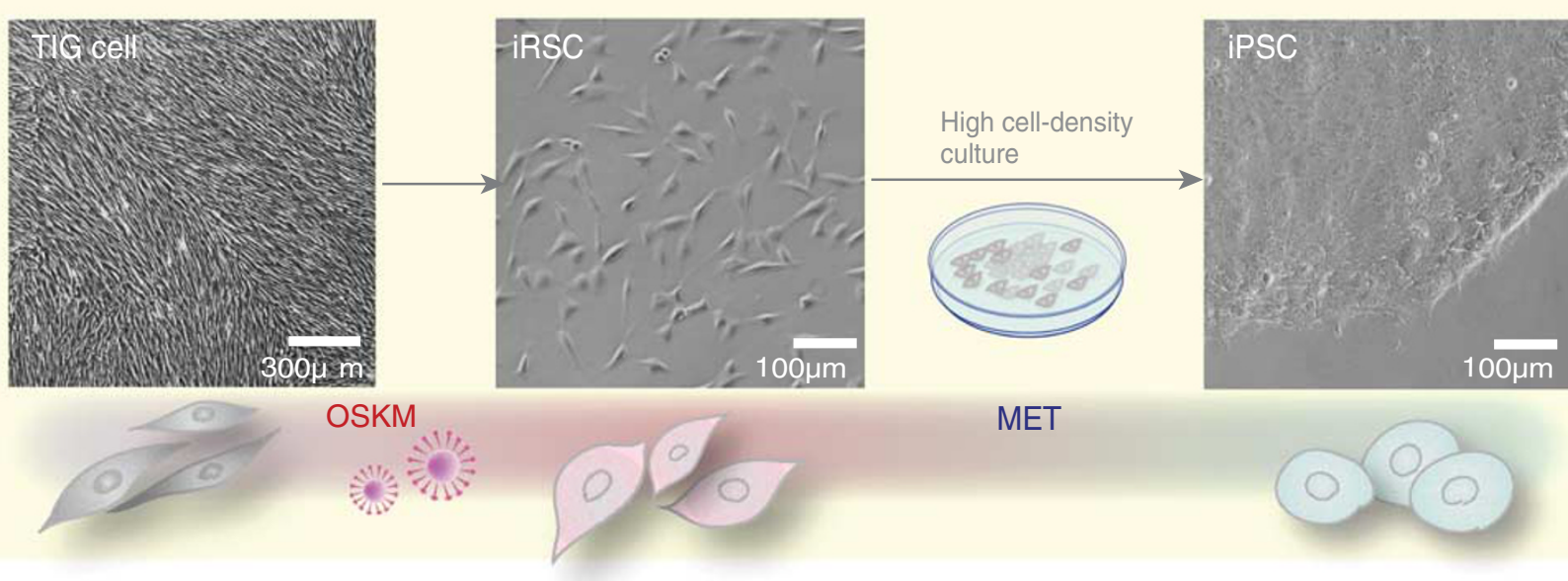

MET

b
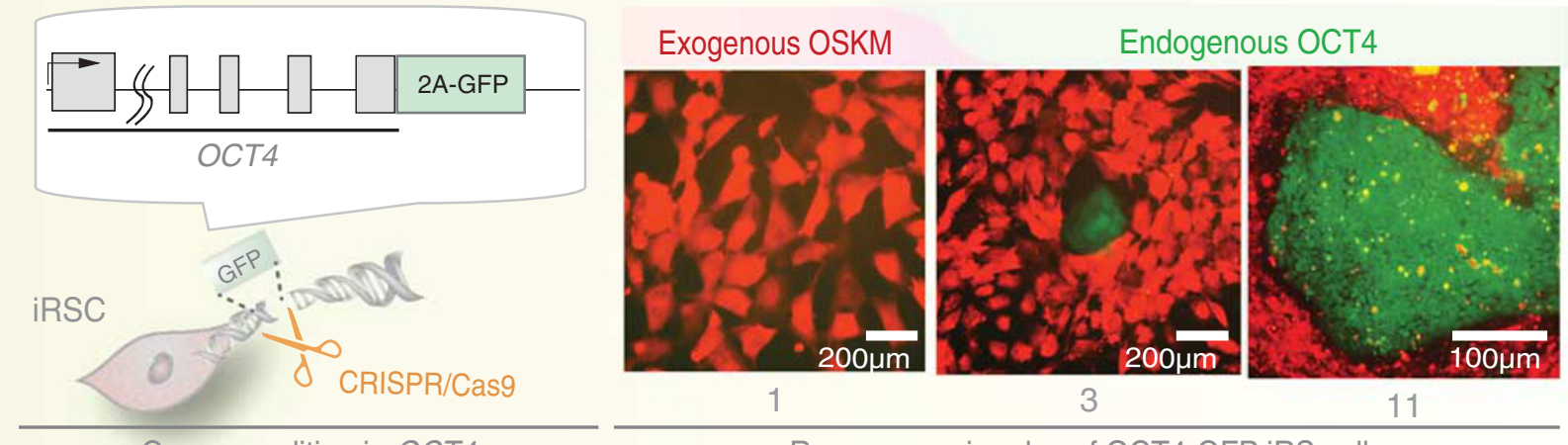

Reprogramming day of OCT4-GFP iRS cells

Figure 2 (a) Sequential changes of cell morphology from human TIG fibroblast cell, intermediately reprogrammed stem cell (iRSC), to induced pluripotent stem cell (iPSC) in somatic reprogramming. OSKM; reprogramming factor, Oct4, Sox2, KIf4, and c-Myc. (b) CRISPR/Cas9-mediated genome editing of the endogenous OCT4 gene in human iRSCs to visualize the activity of OCT4 by fluorescence marker, GFP (Green Fluorescence Protein) in the reprogramming process of iRSCs to iPSCs. MET; mesenchymal-to-epithelial transition. 
characterized by expression of reprogramming transgenes, activated proliferation genes, silencing of pluripotency genes, and aberrant expressing lineage genes were established as several endogenous Oct4-negative pre-iPSC lines without potential of conversion to iPSCs. ${ }^{2,47,48}$ In humans, partially reprogrammed iPSCs resumed reprogramming by upregulation of KLF4 ${ }^{49}$ and pre-iPSC-like cell lines were established as cancer stem cell lines. ${ }^{50}$ Metabolome profiling demonstrated that human partially reprogrammed iPSCs shared only $74 \%$ similarly expressed metabolites with human ESCs, ${ }^{51}$ indicating that transcriptome of partially reprogrammed stem cells is considerably different from that of ESCs. Therefore, establishment of iPSC lines makes no promise to establish stable lines of human partially reprogrammed iPSCs. Under such a state, derivation of human intermediately reprogrammed stem cells (iRSCs) as stable lines was unexpected.

Human iRSCs, established from the reprogrammed fetal lung fibroblast cell lines, TIG1 and TIG3, by retroviral transduction of OSKM reprogramming factors were characterized by silencing of somatic genes, activated reprogramming transgenes, self-renewal ability, activated SSEA4, and potential of conversion to iPSCs ${ }^{52}$ (Figures $1 \mathrm{~b}$ and 2a). Notably, iRSCs efficiently resumed the reprogramming process toward iPSC generation under specified culture conditions at a high cell density. Molecular stimuli involved in resuming reprogramming from iRSC to iPSC are elusive. It has been reported that high cell density is a potent negative regulator of cell cycle and expression of genes including retrotransposon, ${ }^{53}$ implying that exogenous OSKM genes were silenced as a consequence of high cell densitiy of iRSCs. iRSCs were marked by endogenous expression of core pluripotency factors, SOX2 and NANOG but not OCT4, in addition to exogenous OSKM reprogramming factors. Endogenous OCT4 was activated along with entry to MET and silencing of exogenous transgenes. GFP (green fluorescence protein) knockin to the endogenous OCT4 locus by CRISPR/Cas9-mediated genome editing-enabled visualization of the OCT4 activation kinetics in living reprogramming cells transit from iRSCs to iPSCs (Figure 2b). It was revealed that activation of endogenous OCT4 simultaneously occurring with silencing of exogenous OSKM reprogramming factors is induced prior to entry into MET.

Interestingly, time-lapse analyses of endogenous OCT4 activity demonstrated that OCT4-positive reprogramming cells created OCT4-positive and negative daughter cells through asymmetric cell division soon after OCT4 activation, while OCT4-positive cells enabled symmetric cell division to form two daughter cells with the same pluripotent identity in larger growing colonies. ${ }^{52}$ It is likely that instability of endogenous OCT4 is linked to the cell characteristics of symmetric or asymmetric division. It has been debated whether reprogramming entails a hierarchic or stochastic process. ${ }^{54}$ Once OSKM factors are silenced and endogenous OCT4 is activated in a stochastic manner, further reprogramming is proposed to progress in a hierarchical manner. ${ }^{12}$ Contrary to this, it was proposed that endogenous Oct4 activation is insufficient for progression of subsequent events in mouse somatic reprogramming. ${ }^{55,56}$ In the maturation process of iRSC-toiPSC conversion, endogenous OCT4 activation is essential for iPSC generation, but not sufficient for determining cell fate to be iPSCs.

It is controversial whether the reprogramming pathway from somatic cell to iPSC is a single pathway. This is linked to hypotheses as to whether the reprogramming process occurs in a hierarchic or stochastic manner. It has been proposed, in mice, that the pluripotency spectrum can encompass multiple, unique cell states, including an alternative somatic reprogramming path to iPSCs through a Nanog-positive transient state, in addition to the preconceived Nanognegative transient state. ${ }^{57,58}$ Collectively, reprogramming mechanisms of cellular reprogramming from somatic cells to iPSCs are more complicated rather than those we expected when OSKM-mediated somatic reprogramming was discovered.

\section{iRSC APPLICATIONS}

Understanding of molecular mechanisms involved in human somatic reprogramming will not directly contribute to curing specific diseases of patients, but will be useful for investigating medical biology, including human embryonic development, anti-aging, cell physiology, and epigenetics. To do so, application of genetic manipulation to human iPSCs, which is desirable for repair of genetic mutations and deficiencies, is one of the crucial approaches. Single-cell sub-cloning is an inevitable process for genetic manipulation. However, dissociation-induced pro-apoptosis takes place in subcultures of iPSCs, ${ }^{59}$ even using the anti-apoptosis molecule, Rhoassociated kinase (ROCK) inhibitor Y-27632. ${ }^{60}$ An advantageous property of iRSC use is that they are readily expandable from a single cell after conventional gene modifications. Afterwards, reprogramming from gene-manipulated iRSCs to iPSCs can be feasibly resumed by the change of culture conditions. iRSCs will be a powerful cell source for applying recently developed genome-editing technologies. ${ }^{61,62}$ Furthermore, iRSC-based identification of marker genes modulating different reprogramming stages would greatly facilitate the understanding of epigenetic events that occur at each stage by enabling enrichment of subpopulations of reprogramming cells. Integration of a inducible gene expression/repression control system enabled by genetic modifications with iRSCs could help for exploring genes responsible for conversion of iRSCs to 'naïve' iPSCs in the reprogramming. Only a part of the reprogramming mechanism is understood in humans. Further investigation of mechanisms of somatic reprogramming by developing new technologies, and integration with new scientific fields may shed light on the fundamental question of 'what is life'. 


\section{ACKNOWLEDGMENTS}

We thank Ms Megumi Fukuchi for discussion and help.

\section{DISCLOSURE/CONFLICT OF INTEREST}

The authors declare no conflict of interest.

1. Takahashi K, Yamanaka S. Induction of pluripotent stem cells from mouse embryonic and adult fibroblast cultures by defined factors. Cell 2006;126:663-676.

2. Hochedlinger K, Plath K. Epigenetic reprogramming and induced pluripotency. Development 2009;136:509-523.

3. Takahashi K, Okita K, Nakagawa M, et al. Induction of pluripotent stem cells from fibroblast cultures. Nat Protoc 2007;2:3081-3089.

4. Lowry WE, Richter L, Yachechko R, et al. Generation of human induced pluripotent stem cells from dermal fibroblasts. Proc Natl Acad Sci USA 2008:105:2883-2888.

5. Hussein SM, Nagy AA. Progress made in the reprogramming field: new factors, new strategies and a new outlook. Curr Opin Genet Dev 2012;22:435-443.

6. Zhou H, Wu S, Joo JY, et al. Generation of induced pluripotent stem cells using recombinant proteins. Cell Stem Cell 2009;4:381-384.

7. Li $X$, Zhang P, Wei $C$, et al. Generation of pluripotent stem cells via protein transduction. Int J Dev Biol 2014:58:21-27.

8. Zhou YY, Zeng F. Integration-free methods for generating induced pluripotent stem cells. Genomics Proteomics Bioinformatics 2013;11: 284-287.

9. Singh VK, Kumar $\mathrm{N}$, Kalsan $\mathrm{M}$, et al. Mechanism of induction: induced pluripotent. J Stem Cells 2015;10:43-62.

10. Schlaeger TM, Daheron L, Brickler TR, et al. A comparison of nonintegrating reprogramming methods. Nat Biotechnol 2015;33:58-63.

11. Higuchi A, Ling QD, Munusamy MA, et al. Generation of pluripotent stem cells without the use of genetic material. Lab Invest 2015;95: 26-42.

12. Buganim $\mathrm{Y}$, Faddah $\mathrm{DA}$, Cheng $\mathrm{AW}$, et al. Single-cell expression analyses during cellular reprogramming reveal an early stochastic and a late hierarchic phase. Cell 2012;150:1209-1222.

13. Wernig $M$, Zhao JP, Pruszak J, et al. Neurons derived from reprogrammed fibroblasts functionally integrate into the fetal brain and improve symptoms of rats with Parkinson's disease. Proc Natl Acad Sci USA 2008;105:5856-5861.

14. Nagata S, Toyoda M, Yamaguchi S, et al. Efficient reprogramming of human and mouse primary extra-embryonic cells to pluripotent stem cells. Genes Cells 2009;14:1395-1404.

15. Polo JM, Liu S, Figueroa ME, et al. Cell type of origin influences the molecular and functional properties of mouse induced pluripotent stem cells. Nat Biotechnol 2010;28:848-855.

16. Stadtfeld $M$, Hochedlinger K. Induced pluripotency: history, mechanisms, and applications. Genes Dev 2010;24:2239-2263.

17. Brambrink T, Foreman R, Welstead GG, et al. Sequential expression of pluripotency markers during direct reprogramming of mouse somatic cells. Cell Stem Cell 2008;2:151-159.

18. Stadtfeld M, Maherali N, Breault DT, et al. Defining molecular cornerstones during fibroblast to iPS cell reprogramming in mouse. Cell Stem Cell 2008;2:230-240.

19. David L, Polo JM. Phases of reprogramming. Stem Cell Res 2014;12: 754-761.

20. Silva J, Nichols J, Theunissen TW, et al. Nanog is the gateway to the pluripotent ground state. Cell 2009;138:722-737.

21. Polo JM, Anderssen E, Walsh RM, et al. A molecular roadmap of reprogramming somatic cells into iPS cells. Cell 2012;151:1617-1632.

22. Nefzger CM, Alaei S, Knaupp AS et al. Cell surface marker mediated purification of iPS cell intermediates from a reprogrammable mouse model. J Vis Exp 2014;91:e51728.

23. Samavarchi-Tehrani P, Golipour A, David L, et al. Functional genomics reveals a BMP-driven mesenchymal-to-epithelial transition in the initiation of somatic cell reprogramming. Cell Stem Cell 2010;7: 64-77.

24. Esteban MA, Boa X, Zhuang $Q$, et al. The mesenchymal-to-epithelial transition in somatic cell reprogramming. Curr Opin Genet Dev 2012:22:423-428.
25. Leonardo $\mathrm{TR}$, Schultheisz $\mathrm{HL}$, Loring $\mathrm{JF}$, et al. The functions of microRNAs in pluripotency and reprogramming. Nat Cell Biol 2012;14: 1114-1121.

26. Park J, Kwon YW, Ham S, et al. Identification of the early and late responder genes during the generation of induced pluripotent stem cells from mouse fibroblasts. PLoS ONE 2017;12:e171300.

27. Krause MN, Sancho-Martinez I, Izpisua Belmonte JC. Understanding the molecular mechanisms of reprogramming. Biochem Biophys Res Commun 2016;473:693-697.

28. Boward $\mathrm{B}, \mathrm{Wu} \mathrm{T}$, Dalton S. Concise review: control of cell fate through cell cycle and pluripotency networks. Stem Cells 2016;34: 1427-1436.

29. Wang G, Guo X, Hong W, et al. Critical regulation of miR-200/ZEB2 pathway in Oct4/Sox2-induced mesenchymal-to-epithelial transition and induced pluripotent stem cell generation. Proc Natl Acad Sci USA 2013;110:2858-2863.

30. Li R, Liang J, Ni S, et al. A mesenchymal-to-epithelial transition initiates and is required for the nuclear reprogramming of mouse fibroblasts. Cell Stem Cell 2010;7:51-63.

31. Mikkelsen TS, Hanna J, Zhang X, et al. Dissecting direct reprogramming through integrative genomic analysis. Nature 2008:454:49-55.

32. Sridharan R, Plath K. Illuminating the black box of reprogramming. Cell Stem Cell 2008;2:295-297.

33. Polo JM, Hochedlinger K. When fibroblasts MET iPSCs. Cell Stem Cell 2010;7:5-6.

34. Lamouille $S$, Subramanyam D, Blelloch $R$, et al. Regulation of epithelialmesenchymal and mesenchymal-epithelial transitions by microRNAs. Curr Opin Cell Biol 2013;25:200-207.

35. Wang $\mathrm{T}$, Chen K, Zeng $\mathrm{X}$, et al. The histone demethylases Jhdm 1a/1b enhance somatic cell reprogramming in a vitamin-C-dependent manner. Cell Stem Cell 2011;9:575-587.

36. Onder $\Pi$, Kara $N$, Cherry $A$, et al. Chromatin-modifying enzymes as modulators of reprogramming. Nature 2012;483:598-602.

37. Chia NY, Chan YS, Feng B, et al. A genome-wide RNAi screen reveals determinants of human embryonic stem cell identity. Nature 2010;468: 316-320.

38. Hanna J, Cheng AW, Saha K, et al. Human embryonic stem cells with biological and epigenetic characteristics similar to those of mouse ESCs. Proc Natl Acad Sci USA 2010;107:9222-9227.

39. Ying QL, Wray J, Nichols J, et al. The ground state of embryonic stem cell self-renewal. Nature 2008:453:519-523.

40. Hirano K, Nagata S, Yamaguchi S, et al. Human and mouse induced pluripotent stem cells are differentially reprogrammed in response to kinase inhibitors. Stem Cells Dev 2012;21:1287-1298.

41. Pasque V, Plath $\mathrm{K} . \mathrm{X}$ chromosome reactivation in reprogramming and in development. Curr Opin Cell Biol 2015;37:75-83.

42. Gafni $\mathrm{O}$, Weinberger $\mathrm{L}$, Mansour $\mathrm{AA}$, et al. Derivation of novel human ground state naive pluripotent stem cells. Nature 2013;504: 282-286.

43. Guo G, Yang J, Nichols J, et al. Klf4 reverts developmentally programmed restriction of ground state pluripotency. Development 2009;136:1063-1069.

44. Gu KL, Zhang Q, Yan Y, et al. Pluripotency-associated miR-290/302 family of microRNAs promote the dismantling of naive pluripotency. Cell Res 2016:26:350-366.

45. Boyer LA, Lee $\mathrm{TI}$, Cole MF, et al. Core transcriptional regulatory circuitry in human embryonic stem cells. Cell 2005:122:947-956.

46. Collier AJ, Panula SP, Schell JP et al. Comprehensive cell surface protein profiling identifies specific markers of human naive and primed pluripotent states. Cell Stem Cell 2017. e-pub ahead of print 21 March 2017; doi: 10.1016/j.stem.2017.02.014.

47. Theunissen TW, Van Oosten AL, Castelo-Branco G, et al. Nanog overcomes reprogramming barriers and induces pluripotency in minimal conditions. Curr Biol 2011;21:65-71.

48. Chen J, Liu H, Liu J, et al. H3K9 methylation is a barrier during somatic cell reprogramming into iPSCs. Nat Genet 2013;45: $34-42$.

49. Nishimura K, Kato T, Chen $C$, et al. Manipulation of KLF4 expression generates iPSCs paused at successive stages of reprogramming. Stem Cell Rep 2014:3:915-929.

50. Nagata S, Hirano K, Kanemori M, et al. Self-renewal and pluripotency acquired through somatic reprogramming to human cancer stem cells. PLOS ONE 2012;7:e48699. 
51. Park SJ, Lee SA, Prasain N, et al. Metabolome profiling of partial and fully reprogrammed induced pluripotent stem cells. Stem Cells Dev 2017; doi:10.1089/Scd.2016.0320.

52. Teshigawara R, Hirano K, Nagata $\mathrm{S}$, et al. OCT4 activity during conversion of human intermediately reprogrammed stem cells to iPSCs through mesenchymal-epithelial transition. Development 2016;143:15-23.

53. Frisa PS, Jacobberger JW. Cell density related gene expression: SV40 large $\mathrm{T}$ antigen levels in immortalized astrocyte lines. BMC Cell Biol 2002;3:10.

54. Yamanaka S. Elite and stochastic models for induced pluripotent stem cell generation. Nature 2009;460:49-52.

55. Greder LV, Gupta S, Li S, et al. Analysis of endogenous Oct4 activation during induced pluripotent stem cell reprogramming using an inducible Oct4 lineage label. Stem Cells 2012;30:2596-2601.

56. Silva J, Barrandon O, Nichols J, et al. Promotion of reprogramming to ground state pluripotency by signal inhibition. PLoS Biol 2008;6:e253.
57. Tonge PD, Corso AJ, Monetti $\mathrm{C}$, et al. Divergent reprogramming routes lead to alternative stem-cell states. Nature 2014;516: 192-197.

58. Jackson SA, Olufs ZP, Tran KA, et al. Alternative routes to induced pluripotent stem cells revealed by reprogramming of the neural lineage. Stem Cell Rep 2016;6:302-311.

59. Ohgushi M, Sasai Y. Lonely death dance of human pluripotent stem cells: ROCKing between metastable cell states. Trends Cell Biol 2011;21: 274-282.

60. Watanabe $K$, Ueno M, Kamiya D, et al. A ROCK inhibitor permits survival of dissociated human embryonic stem cells. Nat Biotechnol 2007;25: 681-686.

61. Hockemeyer D, Jaenisch R. Induced pluripotent stem cells meet genome editing. Cell Stem Cell 2016;18:573-586.

62. Waddington SN, Privolizzi R, Karda $\mathrm{R}$, et al. A Broad overview and review of CRISPR-Cas technology and stem cells. Curr Stem Cell Rep 2016;2:9-20. 